\title{
The Histopathology of Cystoid Macular Edema*
}

\section{J. Reimer Wolter}

The Departments of Ophthalmology and Pathology of the University of Michigan Medical Center, Ann Arbor, Michigan, USA

\begin{abstract}
The pathological findings in ten eyes with cystoid macular edema are used to demonstrate typical details, stages and clinical associations. A history of ocular hypotony leads the list of associated conditions. Cyclitis is also very common. Separation of the central cones from the pigment epithelium due to folding of the outer retinal layers is observed and this can be used to explain early central vision loss associated with cystoid macular edema.
\end{abstract}

Zusammenfassung. Histologische Befunde in zehn Augen mit cystoider Maculopathy werden zur Demonstration von typischen Details, Entwicklungsstufen und Associationen benutzt. Eine Vorgeschichte von okulärer Hypotonie ist die häufigste Association; aber chronische Cyclitis wird auch oft beobachtet. Bildung einer vertikalen Falte der äußeren Netzhautschichten im Zentrum mit Ablösung der Zäpfchen bietet sich als Erklärung für den Sehverlust in den frühen Phasen von cystoider Maculopathie an.

Cystoid macular edema (CME) has become very important to all ophthalmologists because it may occur after intraocular surgery and spoil otherwise perfect surgical results. Much progress has been made in the clinical description of CME, but as yet its cause remains unexplained. Very little is known about the histopathology of CME. This is largly due to the fact that CME is never a reason for enucleation and, thus, one has to rely on more or less accidental histological observations in eyes that have been removed for other reasons.

Hogan and Zimmerman [1] have provided an excellent histologic view of one stage of rather progressed CME in their basic book on Ophthalmic Pathology (p. 475, Fig. 403,2). This shows the typical features of the condition in its

\footnotetext{
* Supported by The Research To Prevent Blindness, Inc., New York, N.Y.

Offprint requests to: J. Reimer Wolter, M.D., Department of Ophthalmology, University of Michigan Medical Center, Ann Arbor, Michigan 48109, USA
} 
advanced stage: swelling of the central retina with coarse folds of the inner limiting membrane, preservation of a foveolar pit, relatively normal appearance of nerve fiber, ganglion cell and inner plexiform layers, cystoid degeneration in Henle's fiber and inner nuclear layers as well as localized detachment of the very center of the outer limiting membrane with central cones from the pigment epithelium forming a reversed pit or fold. The cystoid change is most advanced in Henle's fiber layer and most of the thickening of the central retina is also due to enlargement of this layer with Henle's fibers "standing up" instead of being in their normal flat position. It is important for differential diagnostic purposes to emphasize that the cystoid spaces in the outer retinal layers appear empty in paraffin sections after routine staining. This view of CME was published in 1962 before the clinical nature of this condition was understood and, thus, it was classified as "cystoid degeneration in the maculas of older persons" and listed under "aging".

With the aim to satisfy the urgent need for a description of the histopathological picture of $\mathrm{CME}$, the present paper describes the stages, the details and the variations of this central retinal process in a number of selected cases. These observations do not as yet allow for a total understanding of the pathogenesis of CME, but they are a step in the right direction. More studies of this kind on the background of clinical observations and experimental work will eventually lead to complete insight.

\section{Case Reports}

Case 1

This is the case of a 57 year-old female who had a 30 year history of orbital extensions of basal cell carcinoma. In 1961 the neoplasm had extended beyond the limits of the orbit and exenteration of the orbital contents with removal of surrounding bones was done as a last attempt to control the neoplasm. The eye was proptosed and vision was very poor at the time of the removal of the eye - due to exposure and corneal scarring. Clinically, the eye ground could not be inspected. At surgery the eye was enucleated first and fixed in formalin immediately. Except for the corneal scarring it was externally normal. Cataract and distinct papilledema were seen grossly after the eye was opened in a horizontal plane. Half of the eyeball was embedded in paraffin and sections were cut and stained with $\mathrm{H}$ and E. The corneal vascularization and scarring as well as the cataract were confirmed histologically. The remainder of the eye was normal except for some peripheral atrophy of the inner retinal layers. The posterior pole with fovea and optic nerve was isolated, cut in frozen sections and stained with several variations of the silver method of Del Rio Hortega.

Silver stains revealed details typical of chronic papilledema with dilation of the veins, swelling and protrusion of the optic nerve bundles and diffuse nerve degeneration (Fig. 1). This process had pushed the retina away from the disk margin. Cystoid edema was seen in the outer layer on the temporal aspect of the disk (Fig. 1). These cystoid spaces appeared empty in the sections - in contrast to a fixed serous exudate that was seen between retina and pigment epithelium on the temporal aspect of an next to the disk (Fig. 1). Some folding of the outer limiting membrane of the displaced retina next ot the disk was observed.

A horizontal section through the foveolar region revealed virtual absence of the inner limiting membrane, large empty cystoid spaces, degeneration of retinal elements with loss of central Mueller's fibers and focal buckling of the inner limiting membrane in a mild vertical fold running through the center (Fig. 2). In a slightly paracentral section with the inner limiting membrane present (Fig. 3), a distinct vertical fold in the outer limiting membrane was found. This buckling of the outer retinal layers represented a small vertical zone of detachment. In this zone the cones were 


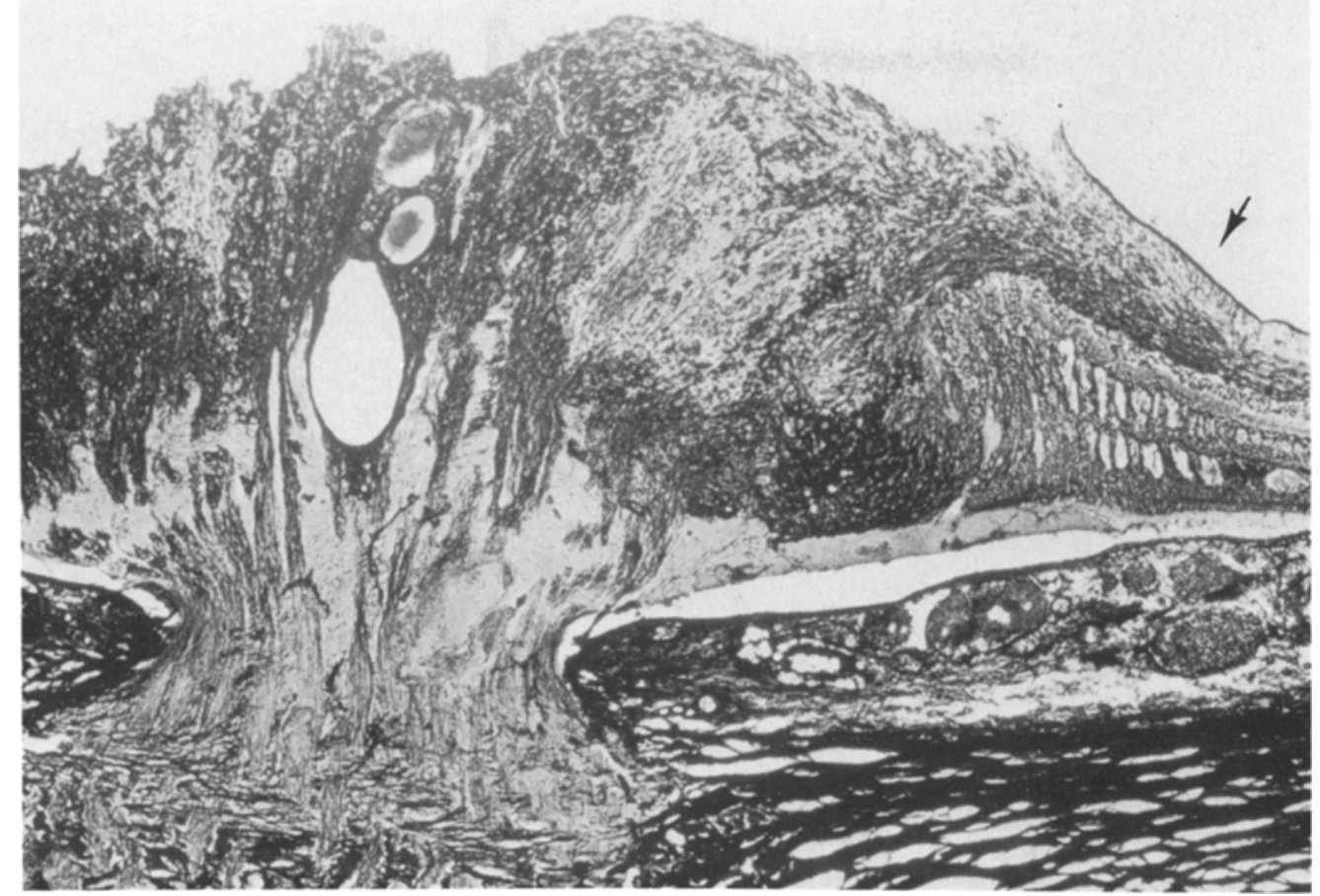

Fig. 1. Case 1, papilledema exhibiting a swollen and prominent disk, cystoid retinal changes temporal to the disk (arrow) and a serous exudate in the retroretinal space. - Horizontal frozen section, Silver stain, photomicrograph $\times 32$

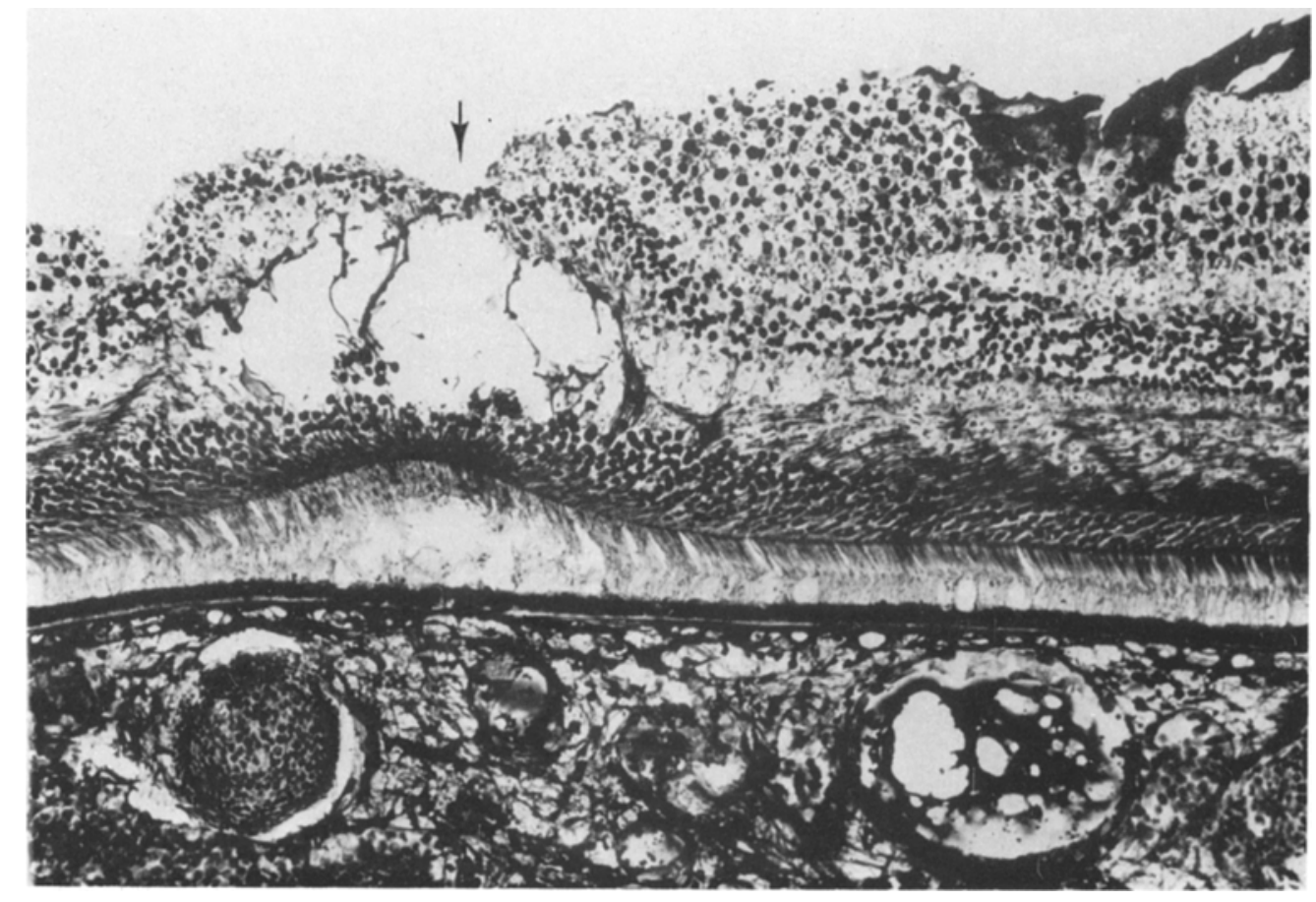

Fig. 2. Case 1, foveola (arrow) with large cystoid spaces, degeneration of neurons and Mueller's fibers as well as separation of the foveolar cones from the pigment epithelium due to a shallow retinal fold. - Horizontal frozen section, Silver stain, photomicrograph $\times 160$ 


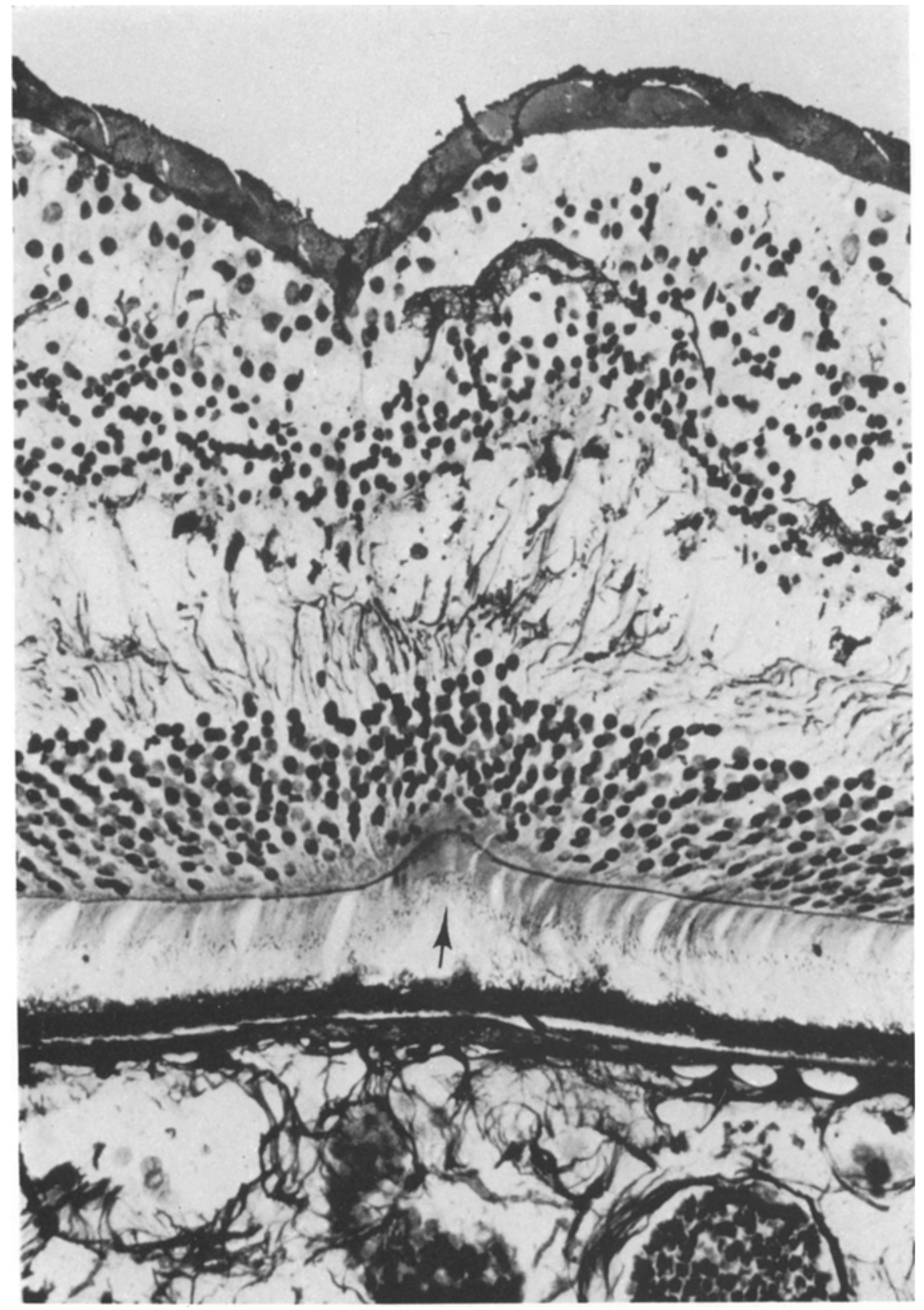

Fig. 3. Case 1, parafoveolar section with continuous inner limiting membrane, cystoid changes as well as thickened and elevated outer limiting membrane forming an outer retinal fold (arrow) of vertical direction. - Horizontal frozen section, Silver stain for glia, photomicrograph $\times 500$ 
no longer in contact with the normal-appearing pigment epithelium. No fixed exudate was found in the space under the retina - in contrast to the subretinal space temporal to the disk with its serous exudate (Fig. 1).

\section{Case 2}

This 57 year-old male had bilateral cataract extractions in 1964 and did very well at first. Vision was 20/20 OU. However, in 1967 a central scotoma developed in the right eye. Associated central retinal changes were seen and all this was classified as "central retinal degeneration". In 1968 bullous keratopathy further complicated the situation and aphakic glaucoma was diagnosed in the same eye. A cyclodialysis with withdrawal of vitreous was done on 9-11-68. The pressure was controlled after that, but the bullous keratopathy continued and a conjunctival flap was done to cover it up on 1-2-70. The eye remained unsightly and painful. It was removed on 3-2-70. Grossly, the eye was of normal size and exhibited corneal scarring, aphakia and partial posterior vitreous detachment. Paraffin sections stained with $\mathrm{H}$ and $\mathrm{E}$ revealed corneal edema, atrophy of the corneal endothelium, chronic non-granulomatous uveitis and diffuse vitreous degeneration.

The gross retinal detachment seen in Fig. 4 is artificial. The section is paracentral and showed the coarse folding of the inner retinal surface that is typical for CME and explains the gross petaloid appearance of this change. The retina was swollen and exhibited cystoid spaces in all layers, the largest in Henle's fiber layer. Some of the cystoid spaces contained a pale-pink exudate without macrophages. The ganglion cell layer was very well preserved. Cystoid macular edema in an advanced stage is diagnosed.

\section{Case 3}

This 86 year-old female had a history of glaucoma and was treated with Pilocarpine and Diamox for at least one year before enucleation. The first recorded examination in the fall of 1973 revealed vision of light perception and a pressure of $58 \mathrm{~mm} . \mathrm{Hg}$. The chamber was shallow. A peripheral iridectomy was done, but the pressure went back up to 60 soon after the operation. The patient asked to have the eye removed and this was done on 9-2-73. The eye was fixed in formalin immediately after enucleation. The eyeball was normal externally. A shallow chamber was seen after the eye was opened. Routine histological examination revealed bullous keratopathy, corneal edema, angle closure, early cataract, chronic non-granulomatous uveitis, posterior vitreous detachment and some diffuse atrophy of the inner layers of the peripheral retina. The optic nerve was cupped and atrophic.

Ganglion cell and nerve fiber layers were somewhat atrophic in the parafoveal retina (Fig. 5). There were many cystoid spaces in Henle's fiber layer and in the inner nuclear layer. These appeared empty in the routine sections, but they were of much smaller size than the spaces of the earlier two cases. Again, the thickening and cystoid change was associated with elevation of the outer retinal layers (inverted pit or shallow fold) and separation of the cones from the pigment epithelium. The central cones were small and decreased in number. The subretinal space did not contain an exudate fixed by formalin and appeared empty. A section through the very center of the foveola was not available in this case. However, the diagnosis of advanced CME could be made.

\section{Case 4}

This 71 year-old female had a large tumor in her left eye that was clinically diagnosed a malignant melanoma. The tumor was located temporal and superior to the fovea. Vision was 20/200. The decreased central vision was explained by subretinal exudate next to the tumor that involved the foveolar region. On 12-9-80 the left eye was enucleated in local anesthesia and the eye was fixed in formalin immediately. Gross examination confirmed the diagnosis of a pigmented tumor in the choroid and this measured $14 \times 11 \times 6 \mathrm{~mm}$. Histological study revealed a shallow anterior chamber with open angle, very early senile cataract as well as slight and diffuse mononuclear infiltration of iris and ciliary body. The retracted vitreous contained exudate that was fixed by formalin. The choroidal tumor had not broken through Bruch's membrane and it had not extended into or through the sclera. It was of a mixed spindle B and epithelioid cell type. The vitreous was detached and retracted.

The foveola seen in horizontal sections was separated from the pigment epithelium by the 


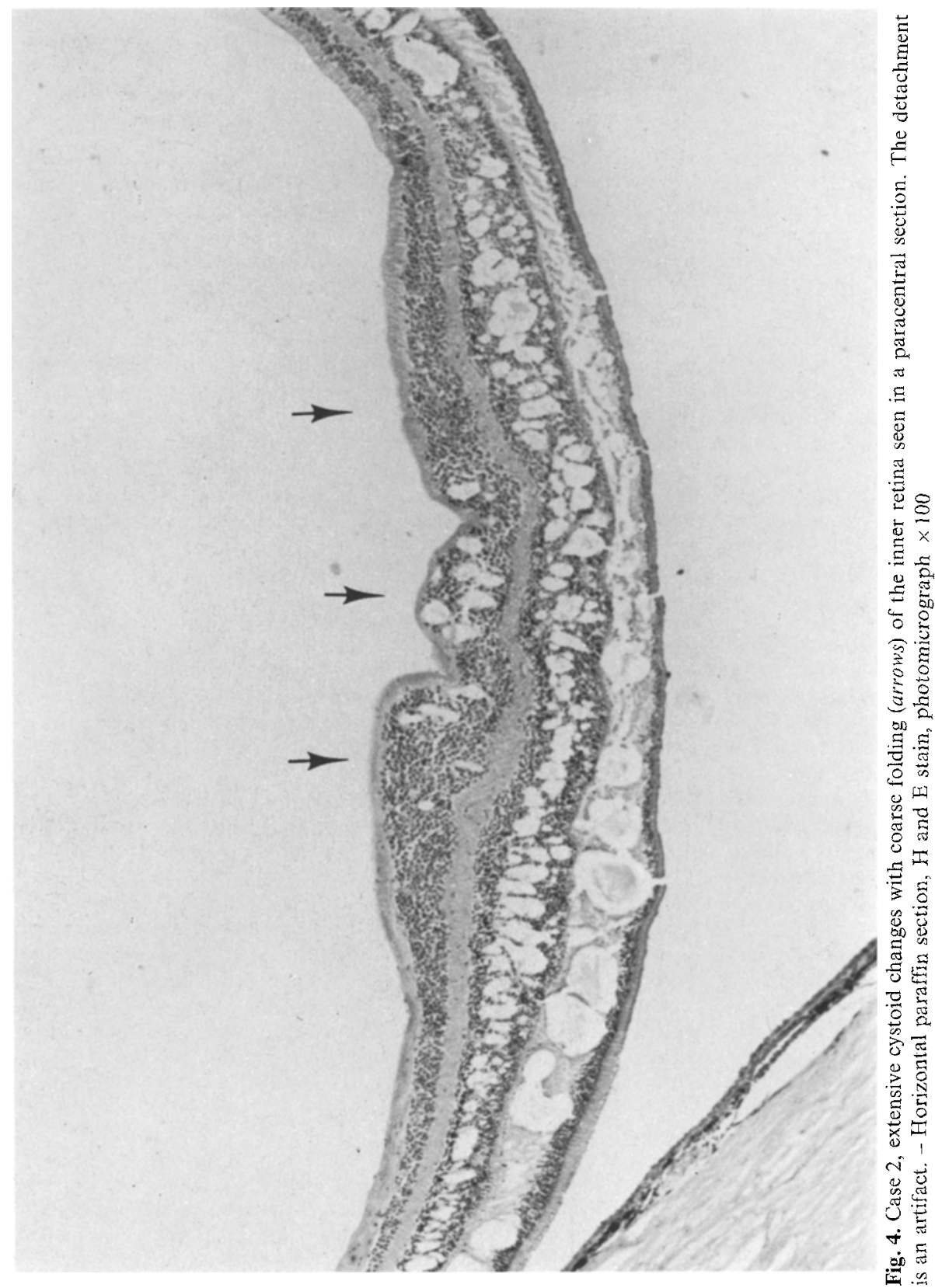

serous exudate (Fig. 6) that surrounded the melanoma. The underlying pigment epithelium was normal, but the choroid exhibited marked vascular congestion explained by the proximity of the malignant tumor. The cones were preserved and surrounded by exudate. Henle's fiber layer was greatly thickened due to fluid accumulation and an early microcystic change in this layer (Fig. 7). Henle's fibers were "standing up" to allow for this swelling. The inner retinal layers were about normal and no connections to the detached posterior hyaloid membrane were seen. A histological diagnosis of a very early stage of CME secondary to a large choroidal melanoma was made. 


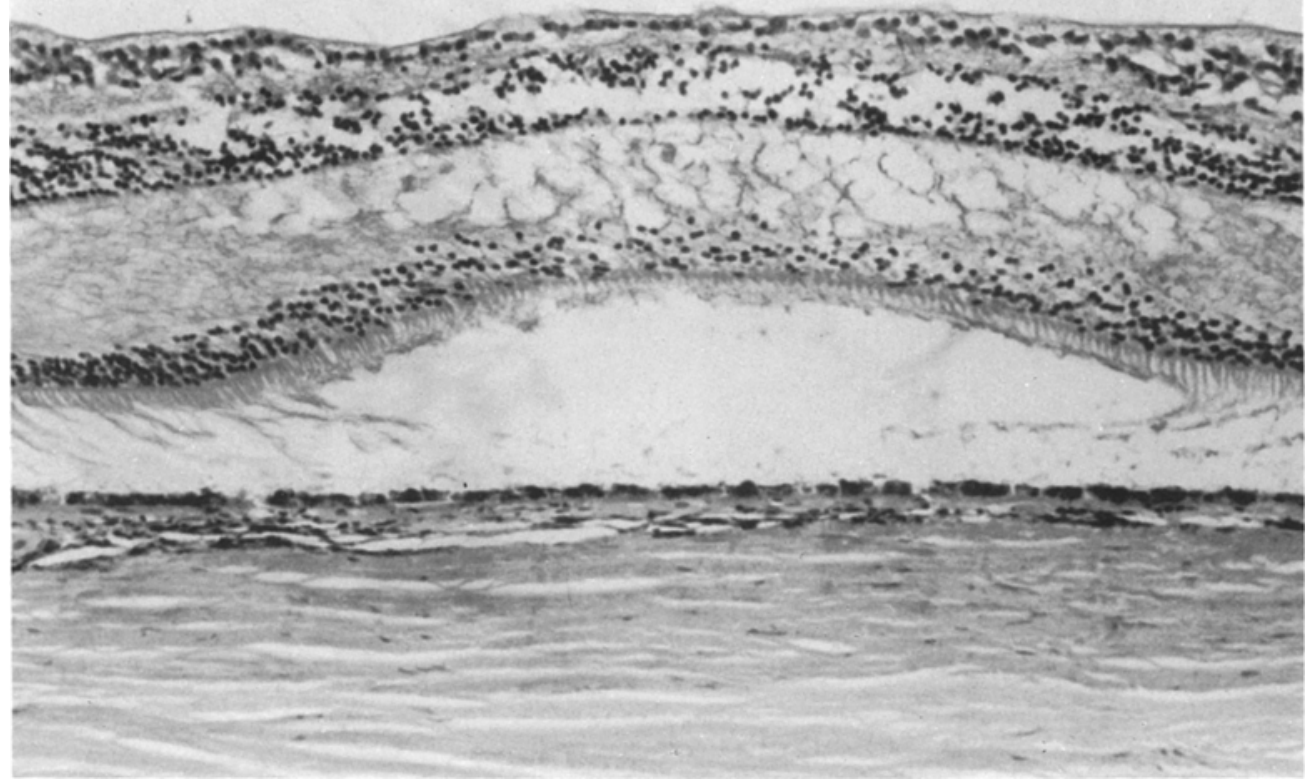

Fig. 5. Case 3, parafoveolar section showing cystoid change with smaller size of cystoid spaces, advanced atrophy of the inner retinal layers and a shallow fold of the outer retina causing detachment of the central cones. - Horizontal paraffin section, $\mathrm{H}$ and $\mathrm{E}$ stain, photomicrograph $\times 120$

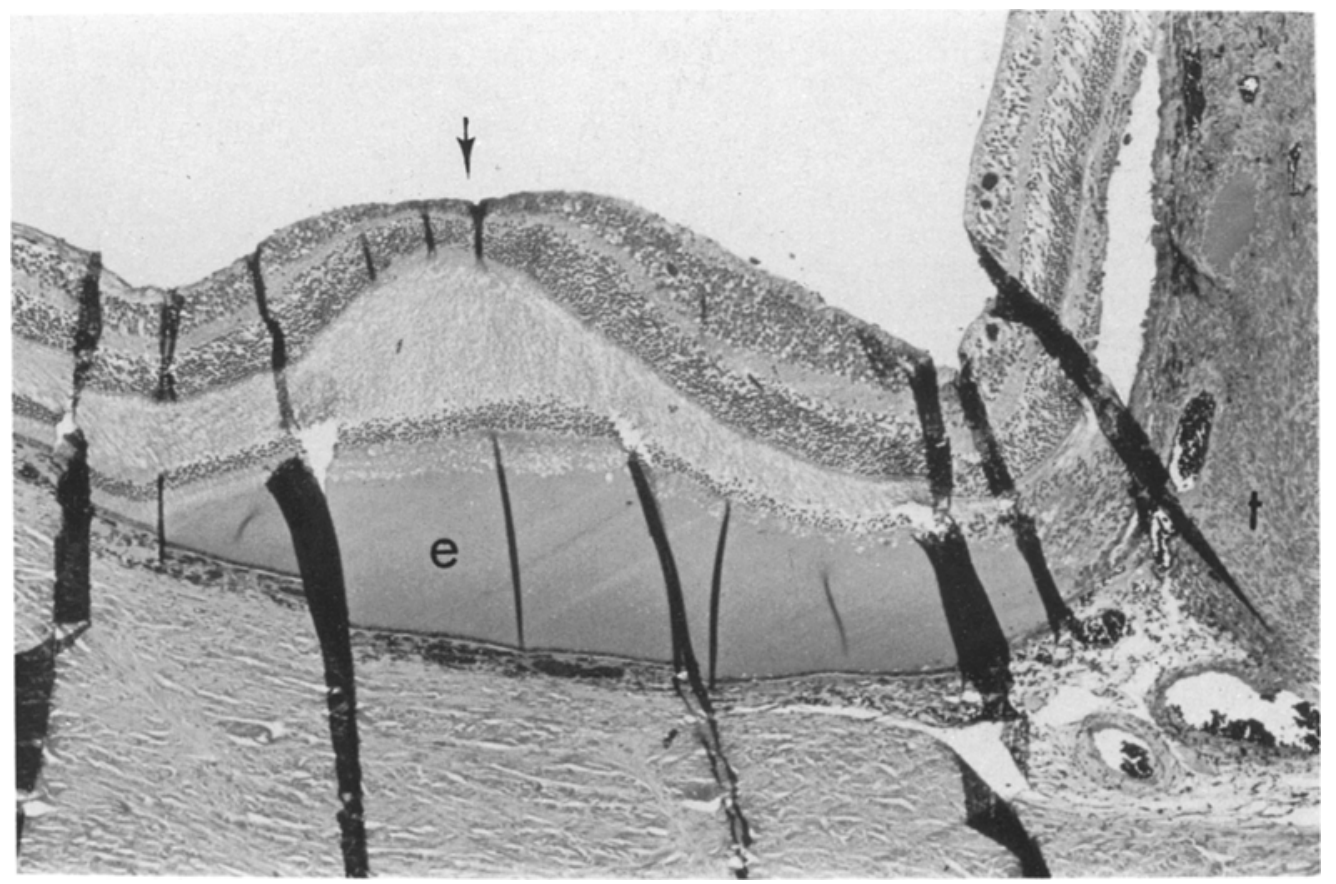

Fig. 6. Case 4, parafoveolar view of swollen Henle's fiber layer (arrow) and retrofoveal serous exudate $(e)$ in choroidal melanoma $(t)$ seen on the right. - Horizontal paraffin section, $H$ and E stain, photomicrograph $\times 40$ 


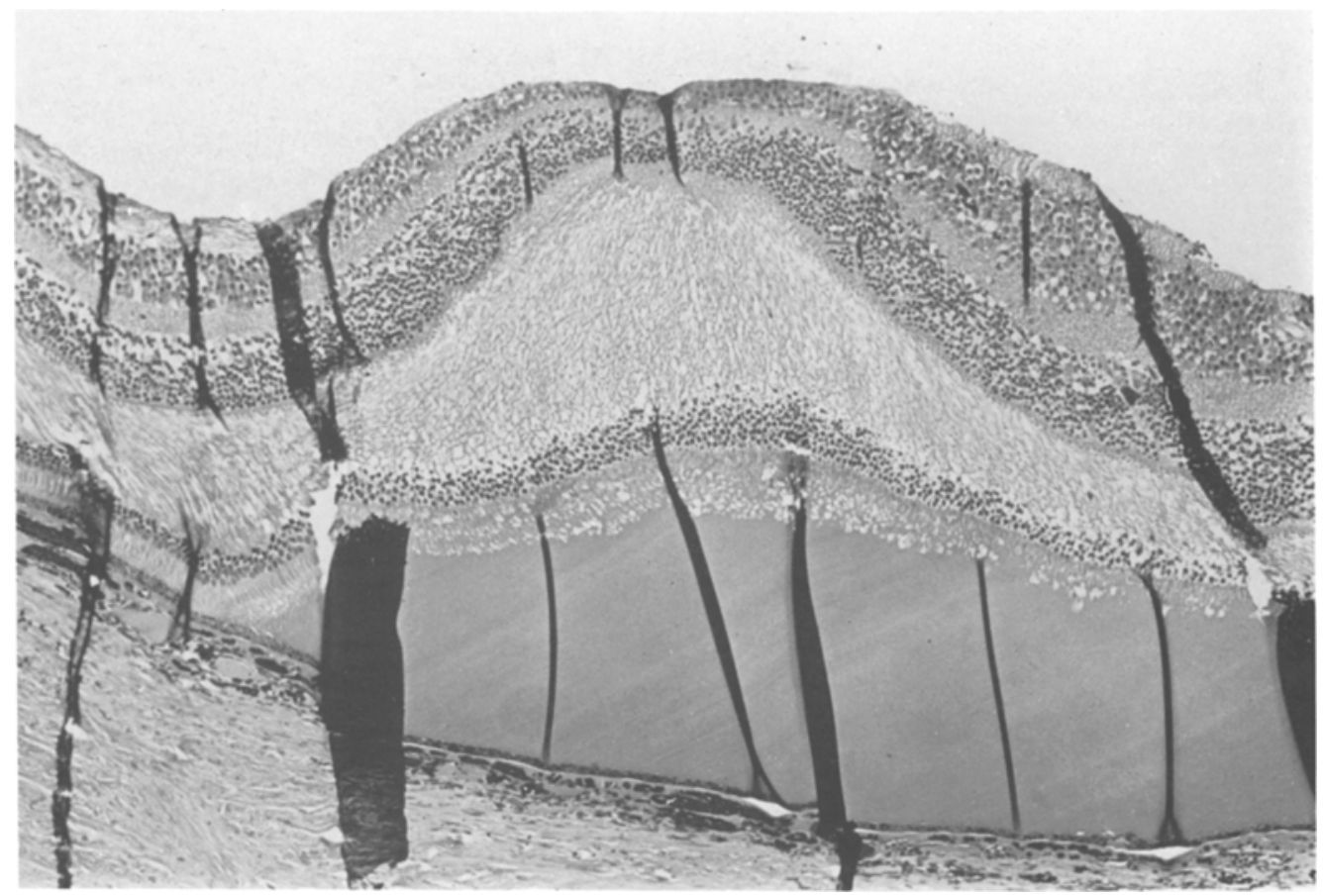

Fig. 7. Case 4, higher power of parallel section to that seen in Fig. 6 to give a better view of the swelling of Henle's fiber layer, early degenerative changes in the detached central cones, the retrofoveal exudate and the well-preserved pigment epithelium in this case. - Horizontal paraffin section, $\mathrm{H}$ and $\mathrm{E}$ stain, photomicrograph $\times 80$

\section{Chase 5}

At the age of almost 97 years this patient had an intracapsular cataract extraction and implantation of an anterior chamber lens of a Choyce type. This was successful and the patient had a visual acuity of $20 / 40$ in the operated eye until he died $2^{1 / 2}$ years later. The eye was donated and could be removed and fixed in formalin about four hours after death. Gross examination of the eye revealed a yellowish-white preretinal exudate and petaloid swelling of the foveal retina. Histological examination of the eye showed inferior impingement of the iris with the haptic of the implanted lens, formation of a callus-like layer of fibrous tissue on the iris surface, slight bleeding next to the vitreous face, a mild chronic uveitis and vitreous retraction. The grossly visible exudate on the posterior retinal surface was firmly attached only to the foveola and was fibrinous in nature.

Sections through the central retina showed extensive swelling with preservation of a foveolar pit (Fig. 8). Remnants of the fibrinous preretinal exudate were recognized in the pit. Coarse folds of the inner retinal layers seen in some of the paracentral sections were the histologic equivalent of the petaloid change seen grossly. The swelling of the retina was most severe in Henle's fiber layer. A distinct microcystic change was recognized in this layer - and this also involved the inner and outer nuclear layers (Fig. 8). In the center of the foveola the outer limiting membrane was thickened and stained poorly. Furthermore, the outer layers were involved in the formation of a horizontal fold of the outer retinal layers with elevation and slight irregular wrinkling of the outer limiting membrane and the cones. The combination of a preretinal exudate with petaloid swelling of the central retina, microcystoid edema and a vertical fold of the outer retina in this case was classified as a mild stage of CME of long duration (comp. Wolter, Croasdale and Bahn [2]). 


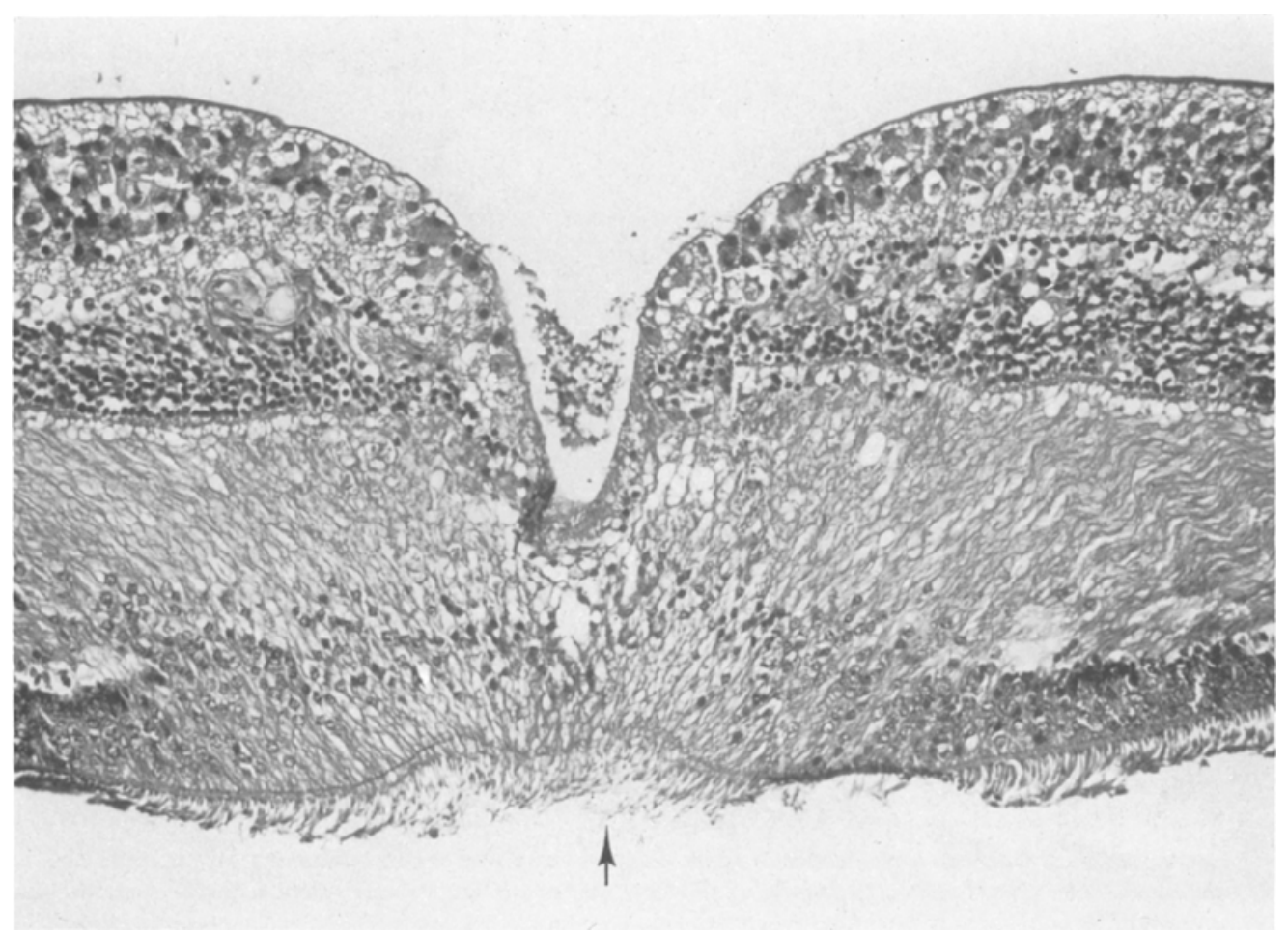

Fig. 8. Case 5, foveolar section showing fibrinous exudate in the internal pit, absence of the inner limiting membrane, edema, microcystic degeneration and a double external fold with elevation and some degneration of the central cones (arrow). - Horizontal paraffin section, H end E stain, photomicrograph $\times 400$

Case 6

This 75 year-old male had a chondroma removed from the right orbit in 1958. This recurred and was again resected in 1967. Regrowth of the tumor occurred for a third time in 1969. On 8-6-69 the right eye had exophthalmus and vision of light perception. On 11-19-69 the right eye was enucleated in the process of more extensive tumor removal from the area of the right orbit.

Grossly the eye was normal in size and exhibited a shallow anterior chamber, papilledema and spotty hemorrhages in the posterior retina. The vitreous was not detached and a layer of vitreous exudate was found to cover the retina in its posterior aspects. Sections revealed a shallow anterior chamber and rubeosis iridis. Early cataractous lens changes were present. There was no posterior vitreous detachment and an eosinophilic exudate of a serous type in the posterior vitreous covered portions of the retina. The disk was distinctly swollen and it also exhibited secondary atrophy.

Advanced CME was found to involve the foveal region (Fig. 9). The foveola was elevated in a vertical fold and an eosinophilic serous exudate had accumulated on the pigment epithelium in the region of this fold (Fig. 9). In addition to the exudate there were pigment-filled macrophages as well as some fibrinous exudate. Large retinal cysts involved Henle's fiber layer, the nuclear layers and even the nerve fiber layer (Fig. 9). The cysts contained some fibrinous exudate and occasional macrophages. Henle's fiber layer also contained localized hemorrhages. Similar hemorrhages were seen posteriorly all over the outer retina. The choroid was congested; its veins were large and blood filled. 


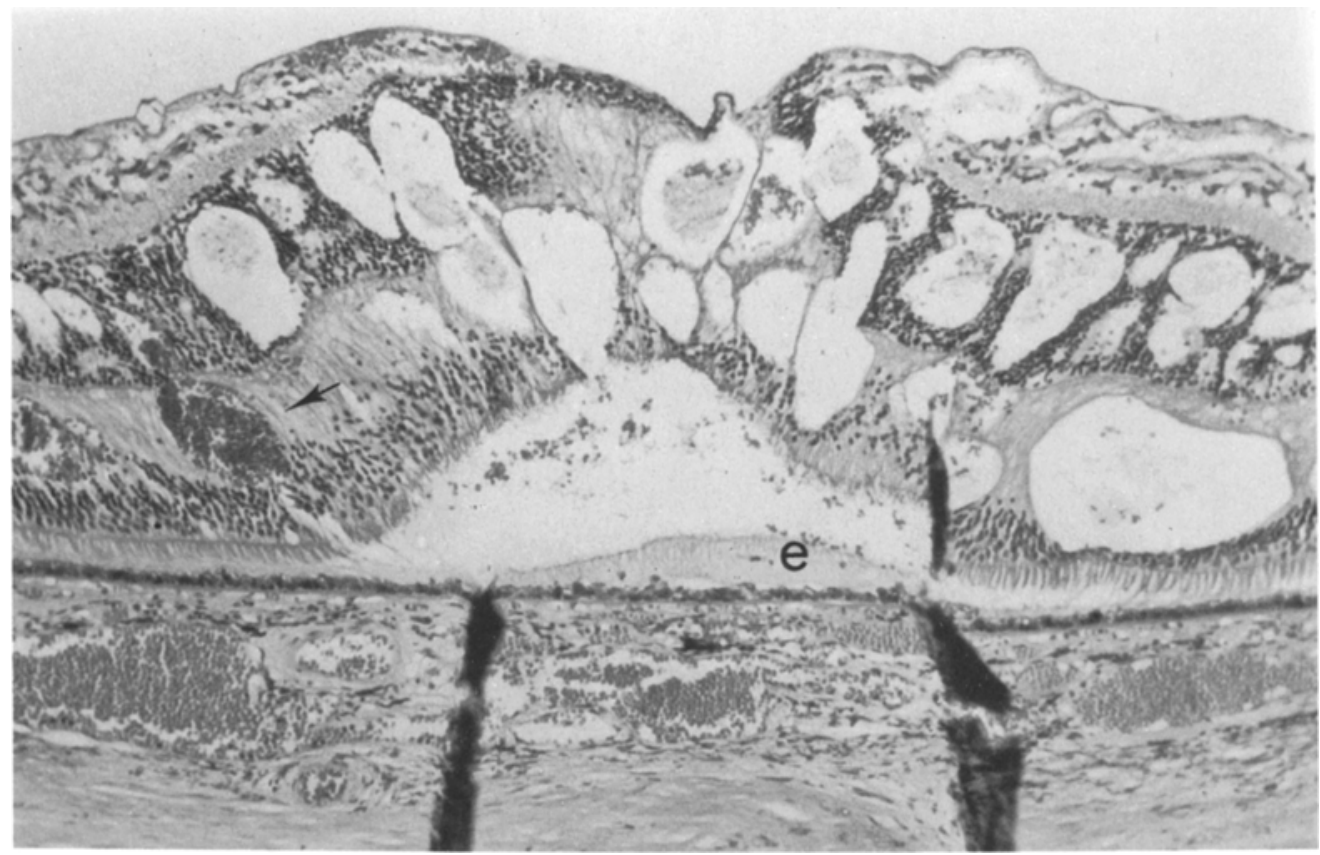

Fig. 9. Case 6, slightly parafoveolar section with many large cystoid spaces containing some fibrinous exudate, a deep retinal hemorrhage (arrow), a retinal fold of the outer retina, central cone degeneration and a shallow serous retrofoveal exudate $(e)$. The choroid is congested. - Horizontal paraffin section, $\mathrm{H}$ and $\mathrm{E}$ stain, photomicrograph $\times 120$

\section{Case 7}

This 67 year-old female had intracapsular cataract extraction and placement of an anterior chamber type of lens implant on 9-19-76. Difficulties developed and the lens implant was removed on 9-8-78. A partial vitrectomy was done along with the implant removal. Phthisis developed and the eye was removed because of pain on $6-6-80$. The eye measured $21 \times 20 \times 19 \mathrm{~mm}$. There was fibrosis in the region of the iris and the retina was totally detached - representing a strand between optic disk and anterior segment. The retroretinal space was filled with a yellow serous exudate that was removed to make sectioning of the eye less difficult.

Histopathological study revealed extensive scarring and neovascularization in back of the cornea. Diffuse mononuclear infiltration was recognized all through iris, ciliary body and choroid. The optic nerve was atrophic and the sclera was thickened. The detached and degenerated retina showed diffuse degeneration and atrophy. A serous exudate filled the central space formed by the detached retina (Fig. 10). An area of thickening with large cystoid spaces indicated the location of the fovea (Fig. 10). The spaces were filled with a very eosinophilic serous exudate. This exudate contained only very occasional macrophages. In spite of the detachment and shrinkage, it was still possible to state that the irregular cystoid spaces were centered on Henle's fiber layer. They were surrounded by extensive gliosis. It was concluded that these large cystoid spaces with exudate were a late stage of CME in a degenerated eye eye with total secondary retinal detachment.

\section{Case 8}

This 22 year-old female had a piece of glass removed from her right eye at the age of 3 years. Gradual loss of vison was observed and the eye, finally, became blind, painful and unsightly. At the age of 22 years, a marked exotropia associated with uveitis and secondary glaucoma was observed. The eye was enucleated on 11-22-67. Gross examination revealed a shallow anterior 


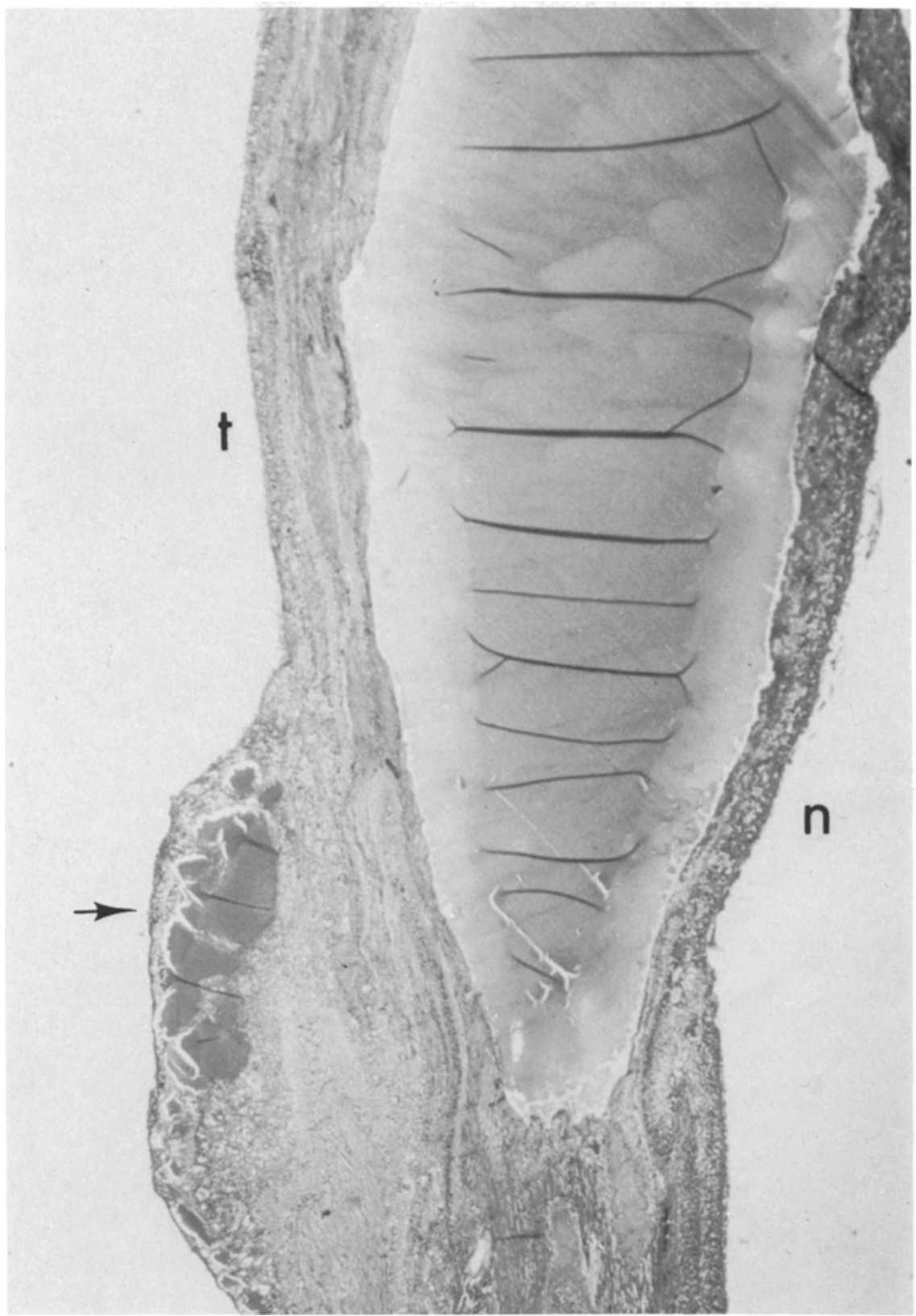

Fig. 10. Case 7, temporal $(t)$ and nasal $(n)$ retina exhibiting extensive detachment with serous exudate in the center. Large cysts with exudate seen in the foveal region (arrow) represent CME. - Horizontal paraffin section, $\mathrm{H}$ and $\mathrm{E}$ stain, photomicrograph $\times 25$ 


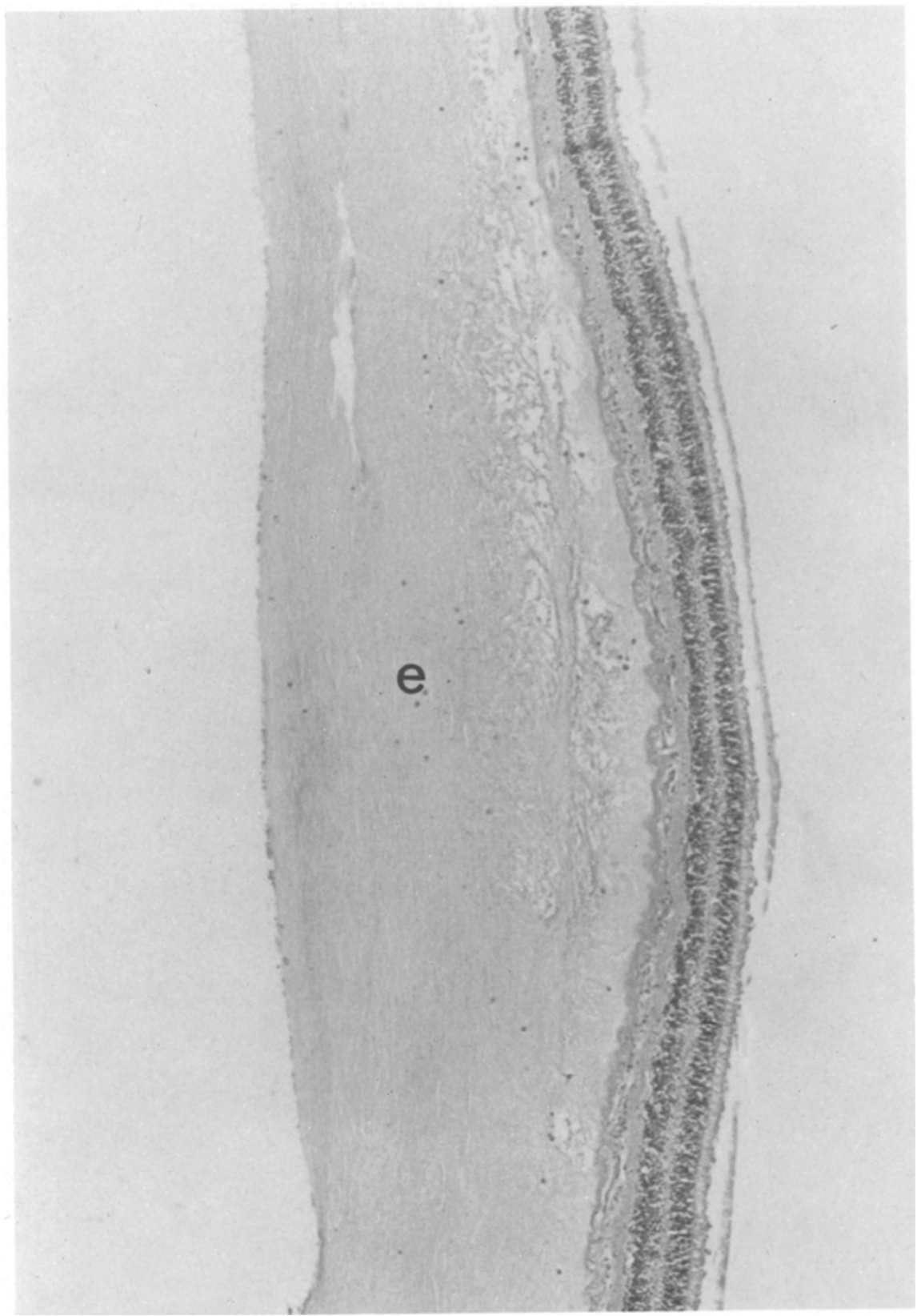

Fig. 11. Case 8, a layer of exudate (e) on the inner surface of the retina in the midperiphery. - Horizontal paraffin section, $\mathrm{H}$ and $\mathrm{E}$ stain, photomicrograph $\times 40$ 


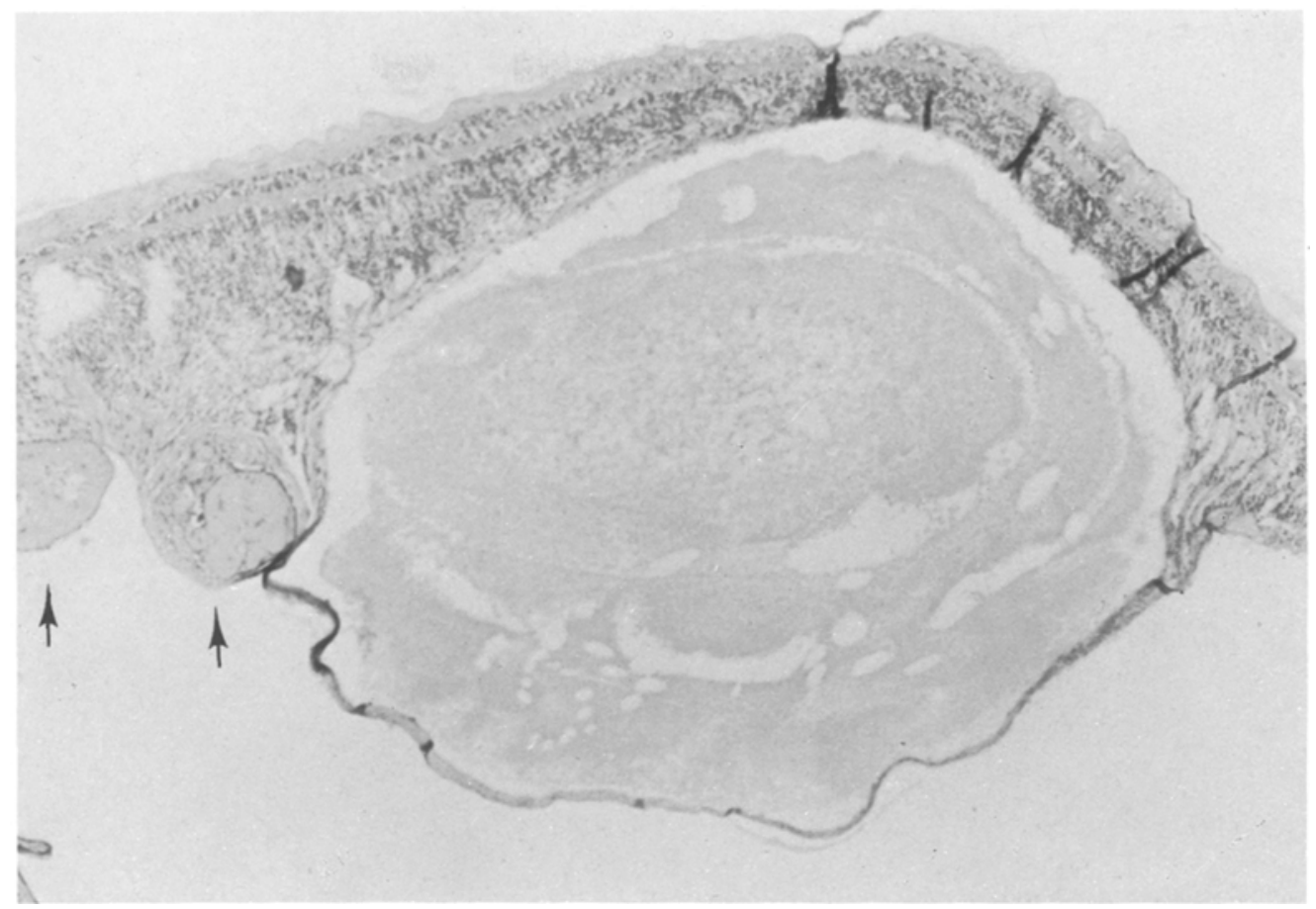

Fig. 12. Case 8, large central retinal cyst centered on Henle's fiber layer filled with a pale serofibrinous exudate. Drusen are seen on the left side of the cyst (arrows). - Horizontal paraffin section, $\mathrm{H}$ and $\mathrm{E}$ stain, photomicrograph $\times 160$

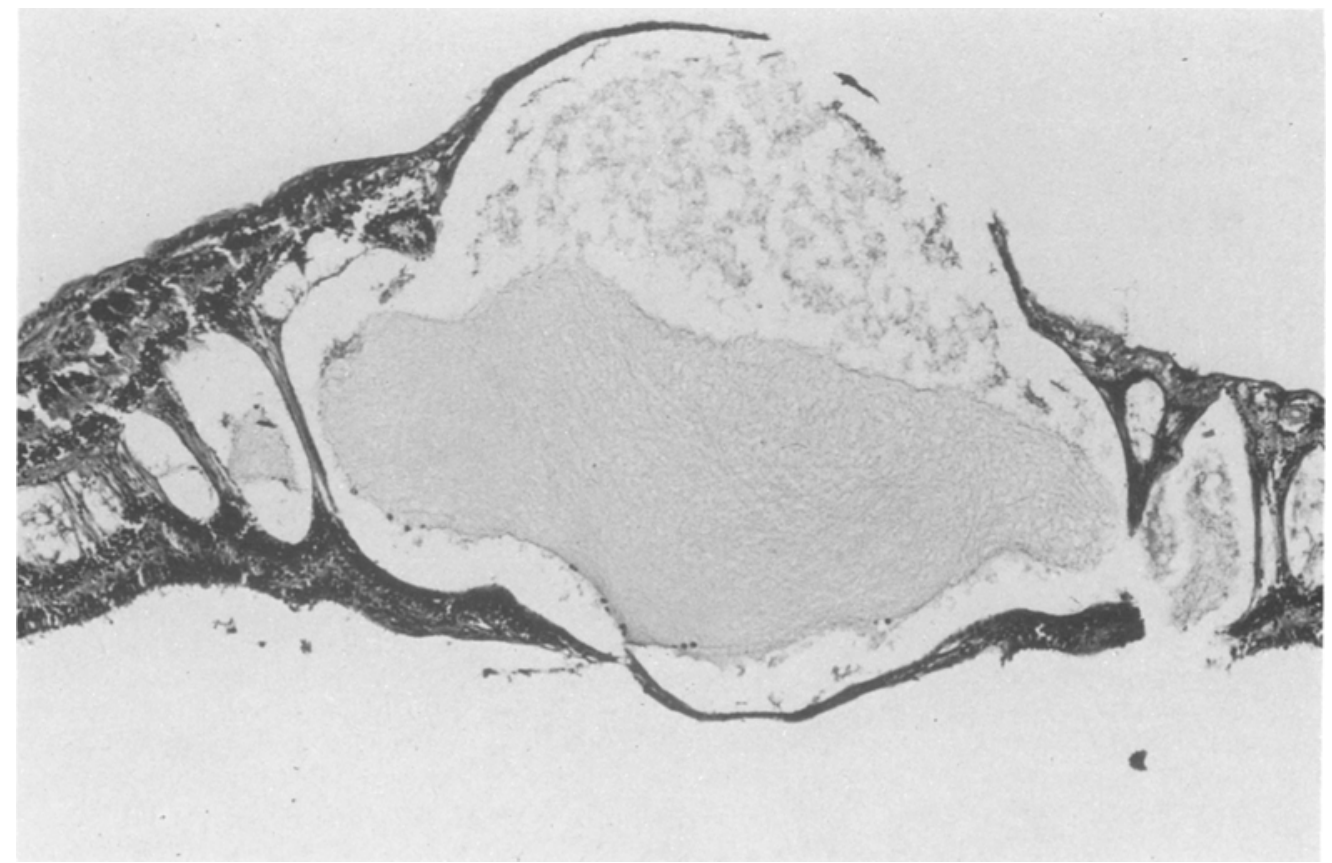

Fig. 13. Case 9, central retinal cyst associated with adjacent smaller cysts in Henle's fiber layer - all filled with a pale and fibrinous exudate. - Horizontal paraffin section, $\mathrm{H}$ and $\mathrm{E}$ stain, photomicrograph $\times 160$ 


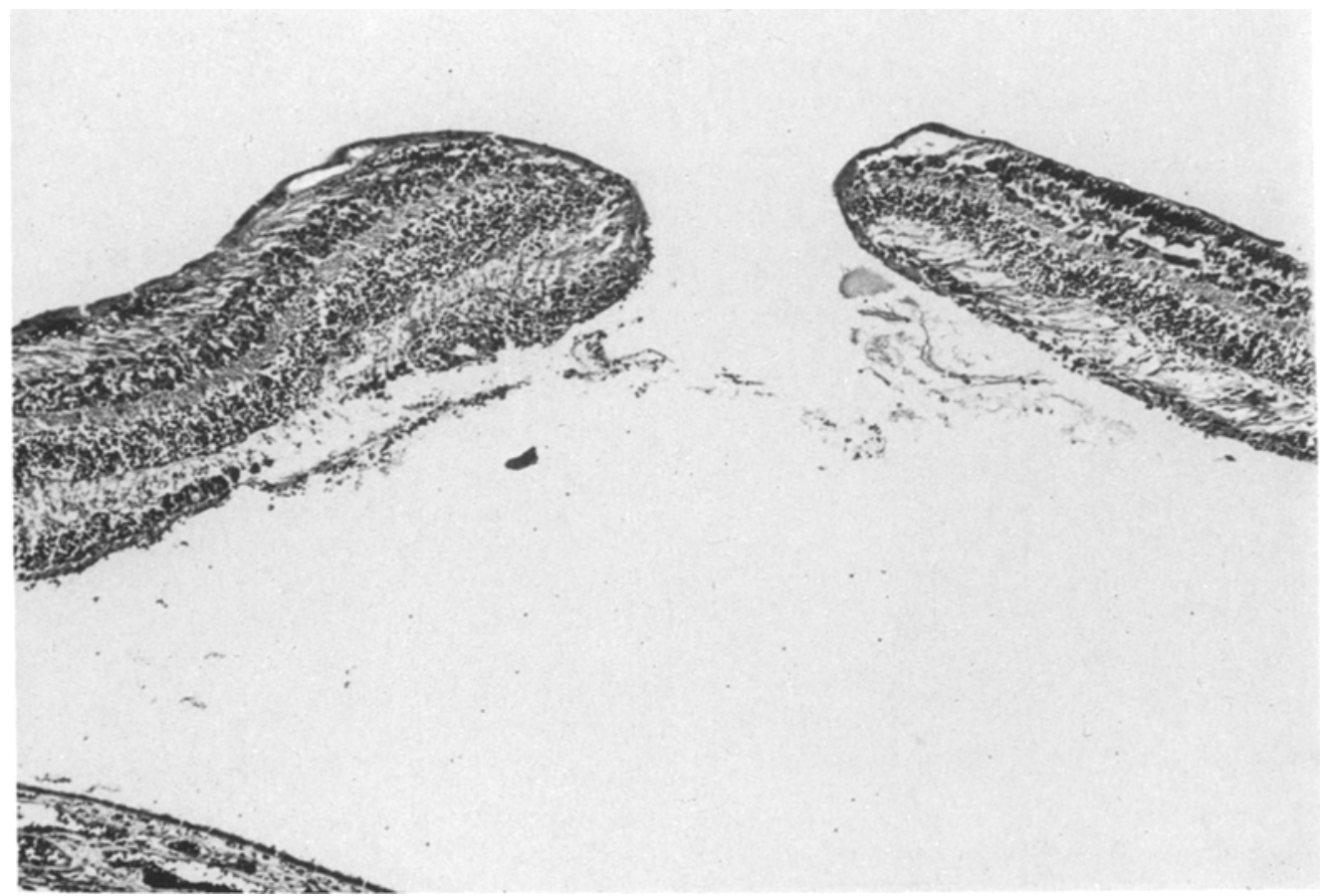

Fig. 14. Case 10, central retinal hole with cystoid changes in Henle's fiber layer on both sides of the hole. The detachment is artificial. - Horizontal paraffin section, $\mathrm{H}$ and $\mathrm{E}$ stain, photomicrograph $\times 160$

chamber, cataract and shallow retinal detachment with a layer of whitish exudate within the vitreous on the inner retinal surface.

Microscopically, bullous keratopathy and angle closure with proliferation of corneal endothelium onto the iris surface were found. The lens was cataractous. Iris and ciliary body exhibited lymphocytes, plasma cells and plasmacytoid cells diffusely. The vitreous showed degenerative changes and a layer of mixed serofibrinous exudate covering large parts of the detached retina (Fig. 11). The shallow retroretinal space appeared empty on the sections. There was some optic nerve atrophy. The central retina exhibited one large cystoid space filled with a pale eosinophilic exudate resembling the preretinal exudate (Fig. 12). The exudate in the cystoid space did not exhibit the large macrophages which are typically seen in "star figures" and "deep hard exudates" of the central retina. The cystoid space centered on a large defect in Henle's fiber layer and also involved the adjacent inner and outer nuclear layers. This cystoid formation was classified as a very late result of longstanding CME.

\section{Case 9}

This 78 year-old female was operated for a cataract in the left eye in 1975 , but she never obtained good vision in the eye. On 12-17-76 she exhibited a red eye with corneal edema and severe bullous keratophathy, corneal edema, atrophy of the corneal endothelium, aphakia, a shallow anterior for enucleation. This was done without difficulties on 1-7-77 and the eye was fixed in formalin immediately. The eye was externally normal and exhibited two iridectomies above. Aphakia and posterior vitreous detachment were recognized when the eye was opened. Histologically, bullous keratophathy, corneal edema, atrophy of the corneal endothelium, aphakia, a shallow anterior chamber with some peripheral anterior synechiae as well as chronic non-granulomatous anterior uveitis with vitreous degeneration and retraction were demonstrated. 
The central retina exhibited one large cystoid space filled with pale fibrinous exudate without large macrophages (Fig. 13). It was not detached and the isolation seen in Fig. 13 is artificial. Large cystoid spaces in Henle's fiber layer seen next to the central space indicated that all this originated from CME and should be classified as a final stage of CME.

Case 10

This 40 year-old male had a severe trauma to his left eye at the age of 14 years. A cataract developed and the eye lost all vision within a few years. Glaucoma complicated the situation and could not be controlled medically. The blind eye became unsightly and caused much pain. It was enucleated on 5-23-80. Grossly, the eye exhibited a shallow anterior chamber and a Soemmering's ring. The retina was grossly seen to be in place. The vitreous was retracted.

Microscopic study revealed corneal edema and the scar of a perforating corneal injury. The angle was closed on one side and open on the other. Diffuse mononuclear infiltration was recognized in iris and ciliary body. The Soemmering's ring exhibited much fibrosis of anterior lens epithelium. Some blood and exudate were accumulated in front of the intact anterior vitreous face. The retina showed advanced atrophy mainly of the inner layers associated with some retinal hemorrhages. In the region of the fovea there was a retinal hole (Fig. 14). Cystoid edema mainly involving Henle's fiber layer was recognized in remnants of the foveal retina surrounding this hole. A late stage of CME was considered the most likely cause of the hole formation.

\section{Discussion}

It is a basic human reaction to take apart any functioning unit that causes problems - with the aim to find the defect. A look at general medical history, thus, reveals that our need to understand a disease process is rarely satisfied without histopathological study and documentation. In Ophthalmology there presently is no other disease process that needs a histopathological foundation more than CME. It is clinically known, however, that CME can occur in association with numerous eye diseases and may well be a typical reaction of the central retina to a great number of different causes. At this point it is best, therefore, to concentrate on detailed histopathological description with consideration of history and associated findings. In view of the lack of good clinical correlations in most cases of this study, speculations are kept at a minimum.

Swelling of the foveal retina is one of the basic components of CME. In the early stages this mainly takes place in Henle's fiber layer - the only layer of the central retina that is built to allow for swelling without known destructive changes [3]. In the process of swelling Henle's fibers "stand up" and, thus, accommodate the accumulation of plasmoid fluid between its fibers (Fig. 7). Small cystoid spaces filled with plasmoid fluid in Henle's fiber layer represent the next stage (Fig. 8). These cystoid spaces are the larger, the more severe the CME becomes (Figs. 7, 8, 9 and 12). With increasing size these cystoid spaces involve the neighboring nuclear layers (Figs. 2, 4, 5 and 9). Associated with progressive size of the cystoid spaces degenerative changes in foveal neurons and glia are found. In mild or early cases of CME the foveola is preserved (Fig. 8). With the development of large cystoid spaces, central Mueller's fibers break down and allow for the central pit to change into a central mound (Fig. 2). Giant spaces, finally, result (Figs. 12 and 13). The remaining layers can break and layer holes may develop. Central retinal holes occur when both, the inner and the outer limiting layers break (Fig. 14). The present histologic 
view of a central retinal hole (Fig. 14) must be a rarity since modern books are printed with only an old drawing made in 1928 to show the histology of this situation $[4,5]$.

The central swelling goes along with the typical petaloid folding of the inner retina. This is grossly visible and I have now seen and photographed this in two cases [2]. This folding of the inner retina appears in parafoveal cross sections as bizarre irregularities (Fig. 4), but this can be reconstructed from serial sections to resemble the grossly known star-shaped formation. The outer retina in CME commonly shows elevation of the central cones in the form of a pit or fold. In the literature there is much unresolved discussion about the significance of the "fovea externa" (reversed pit) seen in sections of "normal" as well as pathologically involved eyes (comp. Apple and Raab [3]). In a recent paper a high retinal fold associated with $\mathrm{CME}$ was demonstrated in an eye after sudden decompression [6]. Our findings indicate that detachment of foveal cones in CME is commonly associated with a fold of the outer retinal layers running vertical in direction. However, all our sections are cut in a horizontal direction. Thus, it would be possible to miss horizontal folding of this type. Michels, Green and Maumenee [7] also reported folding of the outer retina associated with $\mathrm{CME}$ following cataract extraction. Gass [8], finally, saw a high horizontal fold in a parafoveolar section of the retina in a late case of CME with a layer hole. However, in his case the eye was cut vertically and the photographs indicate presence of much autolytic change.

The space between the elevated cones and the pigment epithelium in CME usually appears empty in our sections (Figs. 2, 5 and 8) - and it is believed to be filled with plasmoid fluid. Plasmoid fluid is not fixed by formalin. The elevated cones show degenerative changes - which would explain central vision loss. There also may rarely be an accumulation of a serous or fibrinous exudate in this subretinal space (Fig. 9). The cystoid change of CME is very persistent and can be recognized in very late stages of retinal degeneration and detachment (Fig. 10).

The cystoid spaces in the retina appear empty in early stages of CME (Fig. 5). In later stages exudates may accumulate, but these are never very heavy. They are fibrinous, sero-fibrinous or serous. Typically, the retinal exudates of CME contain very few macrophages - in contrast to the so-called deep hard exudates [9]. Spot-like bleeding in Henle's fiber layer can be associated with the formation of cystoid spaces (Figs. 9). Exudation into the preretinal space is common. It is fibrinous most often (Fig. 8), but it can form homogenous layers and be at least partly serous (Fig. 11). The exudate may be found in the outer parts of the posterior vitreous or in the preretinal space when the vitreous is detached. Commonly, the choroid is thick and congested in cases with CME (Figs. 2 and 9).

There is no need to list all the conditions in which CME has been observed clinically. Review of all my histologically studied cases of CME (unpublished cases and cases published elsewhere [2,6] included) shows that a history of ocular hypotony leads the list of associated conditions - especially when it occurs suddenly and in already irritated eyes. Trauma and surgery are the leading causes of sudden hypotony, of course. Intraocular blood vessels are 
not built to function without the support of the intraocular pressure. Thus, absence of this pressure can be expected to cause a disturbance of the fluid exchange between choroid and retina. Uveitis is next in line - as an associated problem in eyes with CME and cyclitis is the most common type of uveitis. The combination of uveitis and sudden hypotony is the most dangerous and almost always causes CME, it seems. A disturbance of pressure relationships between eye and central nervous system must be important - not only in ocular hypotony, but also in simple papilledema due to increased CNS pressure. Surprisingly, the choroid is commonly thickened and may exhibit both, blood vessel congestion as well as areas of obstruction in the choriocappillaries [6]. CME in melanoma is common, for example, and commonly exhibits greatly increased vascularity and vascular congestion as well as a retroretinal exudate in the foveal zone. Surprisingly, a close association of CME to the conditon of the vitreous is not obvious from histological study. It seems that CME with or without vitreous attachment to the central retina is histologically much the same.

The most important direct conclusion from this study is hat separation of the foveolar cones from the pigment epithelium due to folding of the outer retinal layers frequently occurs in CME. This separation explains central vision loss - and it leads to cone degeneration in the later stages. The separation appears to be an indirect part of the central retinal swelling and it is not difficult to imagine how it can change from day to day and explain the clinically known variations in the vision of patients with CME. If the separation persists, permanent degeneration occurs not only in the layer of the cones, but progressively all through the fovea. Much more speculation would be possible, but it is better to collect more facts. CME is extremely common, both, in the office and in the pathological laboratory.

\section{References}

1. Hogan MD, Zimmerman LE (1962) Ophthalmic Pathology, WB Saunders, Philadelphia, p 475

2. Wolter JR, Croasdale RE, Bahn CF (1980) Reaction to an anterior chamber lens two years after implantation, Ophthalmic Surg, 11:794-800

3. Wolter JR (in print) Concentric "microwaves" of Henle's fiber layer, v Graefe's Arch f Ophth

4. Apple DJ, Raab MF (1978) Clinicopathologic Correlation of Ocular Disease, CV Mosby, St Louis, p 328

5. Apple DJ in Esperance FA jr, James WA (1980) Diabetic Retinopathy, CV Mosby Comp, St Louis, Mo, p 45

6. Wolter JR (in print) Vertical folds of central retina and choroid in sudden ocular decompression, Ophthalmic Surg

7. Michels RG, Green WR, Maumenee A (1971) Cystoid macular edema following cataract extraction, Ophthalmic Surg, 6:217-221

8. Gass JJDM (1975) Lamellar macular hole, a complication of cystoid macular edema after cataract extraction. Transact Amer Ophth Soc, 73:231-248

9. Wolter JR, Goldsmith RI, Phillips RL (1957) Histopathology of the star-figure of the macular area in diabetic and angiospastic retinopathy, Arch Ophth, 57:376-385 